\title{
ON THE EXCURSION PROCESS OF BROWNIAN MOTION
}

\author{
BY
}

FRANK B. KNIGHT ${ }^{1}$

\begin{abstract}
Let $W_{0}^{+}(t)$ denote the scaled excursion process of Brownian motion, and let $l_{0}^{+}(a), 0<a$, be its local time at $a$. The joint distribution of $l_{0}^{+}(a), \beta(a)$, and $\gamma(a)$ is obtained, where $\beta(a)$ and $\gamma(a)$ are the last exit time and the first passage time of $a$ by $W_{0}^{+}(t)$.
\end{abstract}

0. Introduction. In view of the many recent papers on the Brownian meander and excursion (for a partial listing see Durrett, Iglehart, and Miller [4]) it seems almost mandatory for the student of Brownian motion to attempt some contribution in this direction. An unsolved problem of Chung [1, §6] was to find the distribution of the local time of the Brownian excursion at a fixed level $a>0$. This was further investigated in a lengthy paper by Getoor and Sharpe [6], who obtained partial results including limit theorems as $a \rightarrow 0+$, while developing general methods for diffusion functionals. In the present paper, while confining ourselves to the particular situation, we answer this question completely, and meanwhile observe some remarkable and unexpected connections between different features of the excursion process.

A prominent reason for this success is that we depart from the approach of the above authors insofar as they treated the excursion straddling a fixed $t$ (in [6] a Laplace transform over $t$ was then introduced). Instead of this, we treat directly the scaled excursion of [7] and [4], having duration 1. Since an excursion of any fixed duration is equivalent to this by linear changes of scale and time, and since the distribution of the duration of the excursion straddling $t$ is well known by the work of Levy [11], our approach is in this instance the more general.

The scaled excursion was treated in $\$ 2.10$ of Ito and McKean [7], and later by various other authors including [4]. The basis of our method is comprised of just two facts. The first is that the scaled excursion $W_{0}^{+}(t), 0 \leqslant t \leqslant 1$, is the weak limit on $C[0,1]$ as $\varepsilon \rightarrow 0+$ of the standard Brownian motion $W(t)$, conditioned by $W(s)>0,0 \leqslant s \leqslant 1$ and $W(0)=W(1)=\varepsilon$. This is contained in [4, Theorem (5.1)], and for our purposes it may be taken as the definition of the scaled excursion $W_{0}^{+}$. The second fact is that for $a_{i}>0, i=1$ or 2 , and $0<t_{1}<t_{2}<1$, the pro ess $W_{0}^{+}(t), t_{1} \leqslant t \leqslant t_{2}$, conditioned by $W_{0}^{+}\left(t_{i}\right)=a_{i}$, is equivalent in distribution to $W(t)$ conditioned by both $W\left(t_{i}\right)=a_{i}$ and $W(t)>0, t_{1} \leqslant t \leqslant t_{2}$, which in turn is equivalent to the 3-dimensional Bessel process $r(t)$ in $\left(t_{1}, t_{2}\right)$ with $r\left(t_{i}\right)=a_{i}$. Here

Received by the editors August 29, 1978.

AMS (MOS) subject classifications (1970). Primary 60J65, 60J55, $60 \mathrm{~J} 60$.

${ }^{1}$ This work was supported in part by National Science Foundation contract MCS-76-074-71. 
the first equivalence is obvious from our definition, while the second is due to McKean [8] (see also Knight [9, §3]).

1. First entrance and last exit times. The first passage time distribution for the Brownian excursion was obtained by Durrett and Iglehart [3, Proposition (3.3)], but in order to derive the joint density of first entrance and last exit times we need a simpler form not easily obtainable from this. To fix notations, let $\gamma(a)=\inf \{t$ : $\left.W_{0}^{+}(t)=a\right\}$ with inf $\phi=1$, and let $\beta(a)=\sup \left\{t: W_{0}^{+}(t)=a\right\}$ with $\sup \phi=1$. Further, let

$$
F_{1}(x)=\sum_{n=-\infty}^{\infty}(-1)^{n} e^{-n^{2} x}, \quad 0<x<\infty
$$

and

$$
F_{2}(x)=1+2 \sum_{n=1}^{\infty} e^{-n^{2} x}\left(1-2 n^{2} x\right), \quad 0<x<\infty,
$$

with corresponding densities $f_{1}(x)$ and $f_{2}(x)$. It is shown by Chung [1] that these are absolutely continuous probability distributions, and it is remarked that $F_{2}=$ $F_{1} * F_{1}$, where * denotes convolution. For the reader's convenience, a proof of this based on Chung's paper is included at the end of $\S 1$. It is also known from Chung [1] and Kennedy [12] that $F_{2}$ is the distribution of the maximum of $W_{0}^{+}(t)$, hence we have

$$
P\{\gamma(a)=1\}=P\{\beta(a)=1\}=F_{2}(a) .
$$

The first result of the present section is:

THEOREM 1.1. The density of $\gamma(a)$ on $(0,1)$ is

$$
\left(\frac{\pi}{a}\right)^{2}(1-t)^{-3 / 2} \exp \left(-\frac{a^{2}}{2(1-t)}\right) f_{1}\left(\frac{1}{2}\left(\frac{\pi}{a}\right)^{2} t\right)
$$

Proof. We have only to consider the absorption probabilities for $W(t)$ with absorbing barriers at 0 and $a$. For $W(0)=\varepsilon>0$ the absorption probability density at $a$, or outward heat flux, is obtained as $-\frac{1}{2}$ times the derivative at $a$ of the well-known transition density of $W(t)$ with two absorbing barriers [7, §1.7]. Hence it is given by

$$
\frac{\pi}{a^{2}} \sum_{n=1}^{\infty}(-1)^{n+1} n \sin \left(\frac{n \pi \varepsilon}{a}\right) \exp \left(-\frac{n^{2} \pi^{2} t}{2 a^{2}}\right)
$$

The density of probability of $W(1)$ given $W(0)=\varepsilon$ over $\{W(s)>0,0<s<1\}$ is the transition density of $W(s)$ with absorbing barrier at 0 , namely

$$
p_{0}(t, x, y)=\frac{1}{\sqrt{2 \pi t}}\left(\exp \left(-\frac{(x-y)^{2}}{2 t}\right)-\exp \left(-\frac{(x+y)^{2}}{2 t}\right)\right) \text {. }
$$

Hence, formally, to find the probability density of $\{\gamma(a)=t\}$ we need to multiply (1.1) by $p_{0}(1-t, a, \varepsilon)$ and $p_{0}^{-1}(1, \varepsilon, \varepsilon)$ and let $\varepsilon \rightarrow 0+$. As $\varepsilon \rightarrow 0+$ we have 


$$
\begin{aligned}
p_{0}(1-t, a, \varepsilon)= & \frac{1}{\sqrt{2 \pi(1-t)}} \exp \left(-\frac{a^{2}}{2(1-t)}\right)\left(\exp \left(-\left(\frac{-a \varepsilon+\frac{1}{2} \varepsilon^{2}}{1-t}\right)\right)\right) \\
& -\exp \left(-\left(\frac{a \epsilon+1 / 2 \epsilon^{2}}{1-t}\right)\right) \\
= & \frac{1}{\sqrt{2 \pi(1-t)}}\left(\exp \left(\frac{-a^{2}}{2(1-t)}\right)\left(\frac{2 a \varepsilon}{1-t}+o(\varepsilon)\right)\right),
\end{aligned}
$$

and similarly $p_{0}(1, \varepsilon, \varepsilon)=(1 / \sqrt{2 \pi})\left(2 \varepsilon^{2}+o\left(\varepsilon^{2}\right)\right)$. The series $(1.1)$ as $\varepsilon \rightarrow 0+$ is asymptotic to

$$
\frac{\varepsilon \pi^{2}}{a^{3}} \sum_{n=1}^{\infty}(-1)^{n+1} n^{2} \exp \left(-\frac{1}{2}\left(\frac{n \pi}{a}\right)^{2} t\right)
$$

uniformly for $t$ bounded away from 0 . Hence, formally, we obtain the asserted density, uniformly except at $t=0$. This is sufficient to determine the limits of integrals over intervals $\left(t_{1}, 1\right), t_{1}>0$. Finally, it is clear that $I_{\left\{\gamma(a)>t_{1}\right\}}$ is continuous at almost all paths of the Brownian excursion. Hence $P\left\{\gamma(a)>t_{1}\right\}$ is continuous under the weak convergence as $\varepsilon \rightarrow 0+$ and the proof is complete.

We remark that $\mathrm{W}_{0}^{+}$is an inhomogeneous strong Markov process (as follows from the Feller property of the associated space-time process) and $\gamma(a)$ is a stopping time with $W_{0}^{+}(\gamma(a))=a$ on $\{\gamma(a)<1\}$. Hence to obtain the conditional density of $\beta(a)$ given $\gamma(a)=t_{1}$ we can apply the same method as above. Namely, we condition $W(t)$ by $W(t)>0, t_{1}<t<1$, and $W\left(t_{1}\right)=a, W(1)=\varepsilon \rightarrow 0+$. In this way we will obtain

TheOREM 1.2. The conditional density of $\beta(a)$ on $\left(t_{1}, 1\right)$ given $\gamma(a)=t_{1}<1$ is

$$
\frac{1}{2} \frac{\pi^{2}}{a^{4}}\left(1-t_{1}\right)^{3 / 2} \exp \frac{a^{2}}{2\left(1-t_{1}\right)}\left[\frac{1-\exp \left(-2 a^{2} /\left(t-t_{1}\right)\right)}{\sqrt{\left(t-t_{1}\right)}}\right] f_{1}\left(\frac{1}{2}\left(\frac{\pi}{a}\right)^{2}(1-t)\right) \text {. }
$$

Proof. For fixed $\varepsilon>0$, let $\beta\left(t_{1}\right)=\sup \left\{t_{1}<t<1: W(t)=a\right\}$, let $S=\{W(t)>$ $\left.0, t_{1}<t<1\right\}$, and let $P^{(e)}$ denote conditional probability given $W\left(t_{1}\right)=a$ and $W(1)=\varepsilon$ times the density of probability of $W(1)=\varepsilon$ when $W\left(t_{1}\right)=a$. Then

$$
\begin{aligned}
P^{(e)}\left\{\beta\left(t_{1}\right)<t ; S\right\}= & P^{(e)}\{W(s) \neq a, t<s<1 ; S\} \\
= & \frac{1}{\sqrt{2 \pi\left(t-t_{1}\right)}} \int_{0}^{a}\left(\exp \left(-\frac{(y-a)^{2}}{2\left(t-t_{1}\right)}\right)-\exp \left(-\frac{(y+a)^{2}}{2\left(t-t_{1}\right)}\right)\right) \\
& \cdot \frac{2}{a} \sum_{n=1}^{\infty} \sin \frac{n \pi \varepsilon}{a} \sin \frac{n \pi y}{a} \exp \left(-\frac{1}{2}\left(\frac{n \pi}{a}\right)^{2}(1-t)\right) d y
\end{aligned}
$$

in accordance with the known transition densities of $W(t)$ with absorbing barriers at 0 and at $\{0, a\}[7,1.7]$. We divide by

$$
P^{e}(S)=\frac{1}{\sqrt{2 \pi\left(1-t_{1}\right)}}\left(\exp \left(-\frac{(a-\varepsilon)^{2}}{2\left(1-t_{1}\right)}\right)-\exp \left(-\frac{(a+\varepsilon)^{2}}{2\left(1-t_{1}\right)}\right)\right)
$$


and let $\varepsilon \rightarrow 0+$. This term may be replaced by

$$
\frac{1}{\sqrt{2 \pi\left(1-t_{1}\right)}} \exp \left(-\frac{a^{2}}{2\left(1-t_{1}\right)}\right)\left(\frac{2 a \varepsilon}{1-t_{1}}\right),
$$

as seen before, and the series may be simplified by putting $n \pi \varepsilon / a$ for $\sin (n \pi \varepsilon / a)$ as $\varepsilon \rightarrow 0+$, uniformly for $t$ bounded away from 1 . This leads to the limit

$$
\begin{aligned}
& \pi a^{-3}\left(1-t_{1}\right)^{3 / 2}\left(t-t_{1}\right)^{-1 / 2} \exp \left(\frac{a^{2}}{2\left(1-t_{1}\right)}\right) \\
& \cdot \sum_{n=1}^{\infty}\left[n \exp \left(-\frac{1}{2}\left(\frac{n \pi}{a}\right)^{2}(1-t)\right)\right. \\
& \left.\cdot \int_{0}^{a}\left(\exp \left(-\frac{(y-a)^{2}}{2\left(t-t_{1}\right)}\right)-\exp \left(-\frac{(y+a)^{2}}{2\left(t-t_{1}\right)}\right)\right) \sin \frac{n \pi y}{a} d y\right] .
\end{aligned}
$$

Let $I_{n}(t)$ denote the last integral. Two integrations by parts show that

$$
\begin{aligned}
& I_{n}(t)= \frac{a}{n \pi}(-1)^{n+1}\left(1-e^{-2 a^{2} /\left(t-t_{1}\right)}\right)+\left(\frac{a}{n \pi}\right)^{2}\left(t-t_{1}\right)^{-1} \\
& \cdot \int_{0}^{a} \sin \frac{n \pi y}{a}\left(e^{-(y-a)^{2} / 2\left(t-t_{1}\right)}\left(1-\frac{(y-a)^{2}}{\left(t-t_{1}\right)}\right)\right. \\
&\left.-e^{-(y+a)^{2} / 2\left(t-t_{1}\right)}\left(1-\frac{(y+a)^{2}}{\left(t-t_{1}\right)}\right)\right) d y,
\end{aligned}
$$

hence it follows that

$$
\begin{aligned}
I_{n}(t)= & \frac{n \pi\left(t-t_{1}\right) a}{(n \pi)^{2}\left(t-t_{1}\right)-a^{2}}(-1)^{n+1}\left(1-e^{-2 a^{2} /\left(t-t_{1}\right)}\right) \\
& -\frac{2\left(t-t_{1}\right) a^{2}}{(n \pi)^{2}\left(t-t_{1}\right)-a^{2}} \frac{d}{d t} I_{n}(t) .
\end{aligned}
$$

The linear differential equation (1.3) with initial condition $I_{n}\left(t_{1}\right)=0$ is easily solved, and the unique solution is

$$
\begin{aligned}
I_{n}(t)= & \left(\frac{n \pi}{2 a}\right)(-1)^{n+1}\left(t-t_{1}\right)^{1 / 2} \exp \left(-\frac{1}{2}\left(\frac{n \pi}{a}\right)^{2}\left(t-t_{1}\right)\right) \\
& \cdot \int_{t_{1}}^{t}\left(s-t_{1}\right)^{-1 / 2} \exp \left(\frac{1}{2}\left(\frac{n \pi}{a}\right)^{2}\left(s-t_{1}\right)\right)\left(1-e^{-2 a^{2} /\left(s-t_{1}\right)}\right) d s .
\end{aligned}
$$

Substituting back into the series (1.2), the terms in $t$ cancel except for the upper limit in $I_{n}(t)$. It is clear that the derivative in $t$ may be taken term-by-term for $0<t-t_{1}<1-t_{1}$, and this yields the theorem.

Combining Theorems 1.1 and 1.2, we have the following remarkable

COROLlary 1.3. The density on $(0,1)$ of $\beta(a)-\gamma(a)$ is

$$
\pi^{2} a^{-4} t^{-1 / 2}\left(1-\exp -2 a^{2} t^{-1}\right) f_{2}\left(\frac{1}{2}\left(\frac{\pi}{a}\right)^{2}(1-t)\right) .
$$


Proof. Replacing $t-t_{1}$ by $t$ in Theorem 1.2 and integrating times the density of Theorem 1.1 (in the variable $t_{1}$ ) yields

$$
\frac{\pi^{4}}{2} a^{-6} t^{-1 / 2}\left(1-\exp \left(-2 a^{2} t^{-1}\right)\right) \int_{0}^{1-t} f_{1}\left(\frac{1}{2}\left(\frac{\pi}{a}\right)^{2} t_{1}\right) f_{1}\left(\frac{1}{2}\left(\frac{\pi}{a}\right)^{2}\left(1-t-t_{1}\right)\right) d t_{1} \text {. }
$$

Using the fact that $f_{2}=f_{1} * f_{1}$ (as remarked before and proved below) the assertion follows.

REMARK. We have no explanation why the density of the maximum $f_{2}$ should appear again here. The factor $t^{-1 / 2}\left(1-\exp \left(-2 a^{2} t^{-1}\right)\right)$ also has an independent and unexpected meaning, as will be seen in Lemma 2.2.

We include here a proof of the

Convolution identity. $f_{2}(t)=f_{1} * f_{1}(t)$.

Proof. We begin with the identity

$$
f_{1}(t)=\sqrt{2 \pi} \frac{d}{d t}\left(t^{-1 / 2} \sum_{n=-\infty}^{\infty} \exp -\frac{(2 n+1)^{2} \pi^{2}}{4 t}\right)
$$

which follows from the Poisson summation formula (Feller (1966), XIX, 5.10). Here we may take the Laplace transform term-by-term, using the well-known Green function of $W(t)$, to get

$$
\int_{0}^{\infty} e^{-\lambda t} f_{1}(t) d t=\pi \sqrt{2 \lambda} \sum_{n=-\infty}^{\infty} \exp (-\pi \sqrt{\lambda}|2 n+1|) .
$$

Therefore, the transform of $f_{1} * f_{1}$ is

$$
2 \pi^{2} \lambda \sum_{m} \sum_{n} \exp (-\pi \sqrt{\lambda}(|2 n+1|+|2 m+1|))=8 \pi^{2} \lambda \sum_{k=1}^{\infty} k \exp (-\pi \sqrt{\lambda} k) .
$$

Now this may be inverted term-by-term using the first passage time transform

$$
\begin{aligned}
\lambda e^{-\sqrt{2 \lambda} a} & =a \lambda \int_{0}^{\infty} \frac{e^{-\left(\lambda t+a^{2} / 2 t\right)}}{\sqrt{2 \pi t^{3}}} d t \\
& =a \int_{0}^{\infty} e^{-\lambda t} \frac{d}{d t}\left(\frac{e^{-a^{2} / 2 t}}{\sqrt{2 \pi t^{3}}}\right) d t, \quad a>0
\end{aligned}
$$

to get the inverse

$$
4 \sqrt{2} \pi^{3} \sum_{k=1}^{\infty} k^{2} \frac{d}{d t}\left(\frac{\exp \left(-k^{2} \pi^{2} / t\right)}{\sqrt{2 \pi t^{3}}}\right)
$$

Finally, using a theta function identity as in (4.10) of Chung (1976) we have directly

$$
F_{2}(t)=4 \sqrt{2} \pi^{3} \sum_{k=1}^{\infty} k^{2} \frac{\exp \left(-k^{2} \pi^{2} / t\right)}{\sqrt{2 \pi t^{3}}}
$$

proving our assertion.

Caution. Differentiation of the original series for $F_{2}$ gives a series for $f_{2}$ which does not converge uniformly. Hence the above detour seems necessary. 
2. Sojourn and local times. It follows immediately from the joint continuity in $(t, a)$ of the local time of $W(t)$ that the excursion straddling fixed $t$ has a local time continuous in $a$. By rescaling this excursion, we see that the local time

$$
l_{0}^{+}(a)=\lim _{\varepsilon \rightarrow 0} \varepsilon^{-1} \int_{0}^{1} I_{(a, a+\varepsilon)}\left(W_{0}^{+}(t)\right) d t
$$

exists and is continuous in $a$ with probability 1 . It is important to remember that, following [6], we are defining local times relative to Lebesgue measure rather than speed measure. To find the distribution of $l_{0}^{+}(a)$ we will first obtain its conditional distribution given $\beta(a)-\gamma(a)$, and then apply Corollary 1.3. Although $\beta(a)$ is not a stopping time, a condition $\beta(a)=t_{2}$ decomposes into the two conditions $W_{0}^{+}\left(t_{2}\right)$ $=a$, and $W_{0}^{+}(t) \neq a, t_{2}<t \leqslant 1$. The last condition is independent of the process $W_{0}^{+}(t), t \leqslant t_{2}$ given $W_{0}^{+}\left(t_{2}\right)=a$. Since $\gamma(a)$ is a stopping time, we see that if $\gamma(a)=t_{1}$ and $\beta(a)=t_{2}$ are given, $W_{0}^{+}\left(t_{1}+t\right), 0 \leqslant t \leqslant t_{2}-t_{1}$, is simply a Bessel process $r(t)$ with $r(0)=r\left(t_{2}-t_{1}\right)=a$, or equivalently a $W(t)$ conditioned by $W(t)>0$ and $W(0)=W\left(t_{2}-t_{1}\right)=a$. It follows that the conditional distribution of $l_{0}^{+}(a)$ given $\gamma(a), \beta(a)$, and $W_{0}^{+}(t \wedge \gamma(a)), W_{0}^{+}(t \vee \beta(a)), 0<t<1$, is actually dependent only on $\beta(a)-\gamma(a)$.

Suppose, now, that $l_{0}^{+}(a)=\alpha$ is also given. The pair of values $\beta(a)-\gamma(a)$ and $\alpha$ becomes a condition on the process $r(t)$ (starting at $a$ ) which may be stated equivalently as follows: let $T_{\alpha}$ be the time when the local time of $r(t)$ at $a$ reaches $\alpha$ (or $T_{\alpha}=\infty$ if it does not reach $\alpha$ ); then $T_{\alpha}=\beta(a)-\gamma(a)$. Thus to find the distribution of $l_{0}^{+}(a)$ given $\beta(a)-\gamma(a)$ it suffices to find that of $T_{\alpha}$ for all $\alpha$, and then to invert the conditioning by using the known distributions of the total time and the local time of $r(t)$ at its last exit from $a$. In other words, for $r(0)=a$ the conditional distribution of $l_{0}^{+}(a)$ is the same as if we were given that $\beta(a)-\gamma(a)$ is the last exit time of $r(t)$ from $a$. This is clear by decomposing the last exit condition just as was done above for $\beta(a)$.

The formulas of Ito and McKean [7, Chapter 6.2] for the Lévy measures of inverse local times can be adapted to the present situation, although $r(t)$ is transient so that they do not apply directly. But it is easier to appeal directly to the proof of Theorem 2.1 of Knight (1969). According to this, the Laplace transform in $\lambda$ of the total sojourn in $(0, a)$ for $W(t), W(0)=0$, conditioned by $W(t)<a$ up to the time when the local time at 0 reaches $\alpha$ is $\exp \left(\alpha / 2 a-\alpha(\lambda / 2)^{1 / 2} \operatorname{cotanh} a \sqrt{2 \lambda}\right)$. The conditioning makes the Brownian motion into a Bessel process, and interchanging 0 and $a$ we recognize the transform of the sojourn of the present $r(t)$ in $(0, a)$ at time $T_{\alpha}$, conditional upon $T_{\alpha}<\infty$.

REMARK. It is interesting to note that the formula la) of [7, Chapter 6.2.] reduces to the same expression, using Bessel function identities for $I_{1 / 2}$.

Now let us consider the sojourn of $r(t)$ in $(a, \infty)$ before $T_{\alpha}$ given that $T_{\alpha}<\infty$. Then up to time $T_{\alpha}$ the given condition means that we have $r(t)$ conditioned to return to $a$. But if, for some $t_{1}$ we have $r\left(t_{1}\right)>a, t_{1}<T_{\alpha}$, then up to the next return to $a$ this makes $r(t)$ an ordinary $W(t)$. To see this, we note that the conditioned process killed at $a$ is obviously Markov, so we need only compute its transition 
function. Now we use the well-known identity

$$
p_{r}(t, x, y)=p_{0}(t, x, y) y / x
$$

where $p_{r}$ is the transition density of $r, p_{0}$ is the transition density of $W$ killed at 0 , and $y / x=P^{y}(r$ reaches $a) / P^{x}(r$ reaches $a) ; a<x, y$. Then, letting $r_{a}(t)$ denote the process $r(t)$ killed at $a$, we have

$$
\begin{aligned}
& P^{x}\left(r_{a}(t) \in d y \mid r \text { reaches } a\right)=P^{x}(r(s)>a, 0<s<t ; r(t) \in d y) y / x \\
&= P^{x}(W(s)>a, 0<s<t \mid W(t) \in d y ; W(s)>0,0<s<t) \\
& \cdot P^{x}(r(t) \in d y) y / x \\
&= P^{x}(W(s)>a, 0<s<t ; W(t) \in d y) p_{0}^{-1}(t, x, y) p_{r}(t, x, y) y / x \\
&= P^{x}(W(s)>a, 0<s<t ; W(t) \in d y) .
\end{aligned}
$$

It follows that conditional on $T_{\alpha}<\infty$ the sojourn of $r(t)$ in $(a, \infty)$ by time $T_{\alpha}$ is equivalent to that of $W(t), W(0)=a$. In fact, the corresponding Lévy measure for the inverse local time, as constructed in [7, 6.2], depends only on the behavior of the process in $(a, \infty)$, and clearly no time is spent at $a$ itself. But for $W(t)$ the Laplace transform in $\lambda$ of the sojourn in $(a, \infty)$ by $T(\alpha)$ is just $\exp (-\alpha \sqrt{\lambda / 2})$. This is easily seen since, for example, its square $\exp (-\alpha \sqrt{2 \lambda})$ is the transform of $T(\alpha)$ itself for $P^{a}$ (the sojourns in $(-\infty, a)$ and $(a, \infty)$ being independent) and by a famous result of P. Lévy $T(\alpha)$ is equivalent to the passage time from 0 to $\alpha$, which does have this transform.

Finally, it is easy to see from the strong Markov property at times $T_{\alpha}$ that the local time of $r(t)$ at $a$ at the time of last exit from $a$ is exponentially distributed. But its expectation is just the total time integral of the transition density from 0 to $a$, or $(2 a)^{-1}$ by explicit calculation. Hence the density is $(2 a)^{-1} \exp \left(-(2 a)^{-1} \alpha\right), \alpha>0$.

REMARK. One simple but interesting consequence of the last two paragraphs is the transform of the total time spent by $r(t)$ in $(a, \infty)$ up to the last exit time from $a$. Integrating the transform $\exp (-\alpha \sqrt{\lambda / 2})$ times the absolute density of $\alpha$, we have immediately $(1+a \sqrt{2 \lambda})^{-1}$, which means that the sojourn time density is

$$
\left(2 a^{2}\right)^{-1}\left((\pi t)^{-1 / 2}-e^{t} \operatorname{erfc}\left(\frac{t}{2 a^{2}}\right)^{1 / 2}\right), t>0
$$

(Roberts and Kaufman [13, p. 211, \#49]). Moreover, this equals the density of the time spent in $(a, \infty)$ by $W(t), W(0)=a$, up to its first passage to 0 (see for example Lemma 1.1 of Knight (1978)). This identity can be shown directly, using time reversal with respect to the stationary density $x d x$ for $W(t)$ absorbed at $x=0$, and should be compared with identity (1.9) of [6].

We can now easily obtain

LEMMA 2.1. The joint Laplace transform of the last exit time of $r(t)$ from a $>0$ $(r(0)=a)$ and the ultimate local time at $a$, in the transform variables $\lambda$ and $\beta$ respectively, is

$$
(2 a)^{-1}(1-\exp (-2 a \sqrt{2 \lambda}))(\beta(1-\exp (-2 a \sqrt{2 \lambda}))+\sqrt{2 \lambda})^{-1} .
$$


Proof. The sojourns in $(0, a)$ and in $(a, \infty)$ by the last exit time from $a$ are of course conditionally independent if the ultimate local time $\alpha$ at $a$ is given. The last exit condition at $T_{\alpha}$ being, as before, independent of the past, this is just the Markov property in $x$ of the local time at $T_{\alpha}$ given $\left\{T_{\alpha}<\infty\right\}$ (see [9] or [7, 6.2]). Hence we need only multiply the product of the two transforms obtained above times $(2 a)^{-1} \exp \left(-\left(\beta+(2 a)^{-1}\right) \alpha\right)$ and integrate over $\alpha$. The exponentials simplify, and we get

$$
(2 a)^{-1}(\beta+\sqrt{\lambda / 2}(1+\operatorname{cotanh} a \sqrt{2 \lambda}))^{-1}
$$

By routine algebra this reduces to the expression (2.1), which was chosen to facilitate inversion of the transform.

LEMMA 2.2. The joint density of the last exit time and the ultimate local time of Lemma 2.1 , in the variables $t>0$ and $\alpha>0$ respectively, is given by

$$
f(t, \alpha)=-\frac{(2 \pi t)^{-1 / 2}}{2 a} \sum_{\nu=0}^{\infty} \frac{1}{\nu !} \frac{d^{\nu-1}}{d \alpha^{\nu-1}}\left(\alpha^{\nu} \frac{d^{2}}{d \alpha^{2}} \exp \left(-\left(2 t^{-1} a^{2}(\alpha+\nu)^{2}\right)\right)\right),
$$

where $\left(d^{-1} / d \alpha^{-1}\right)(d / d \alpha)=I$.

The marginal density in $t$ is

$$
\frac{(2 \pi t)^{-1 / 2}}{2 a}\left(1-\exp \left(-2 a^{2} t^{-1}\right)\right)
$$

Remarks. Using Leibnitz' rule, the derivatives in (2.2) can be expanded in terms of the Hermite polynomials $H_{1}(x)=x$,

$$
\frac{d}{d x}\left(H_{k}(x) e^{-x^{2} / 2}\right)=-H_{k+1}(x) e^{-x^{2} / 2}, \quad 1<k .
$$

The marginal density in $\alpha$ was noted earlier. The marginal in $t$ also occurred earlier as a factor in Corollary 1.3, for unknown reasons.

Proof. The problem is to invert (2.1). Setting $\sqrt{2 \lambda}=\mu$ we first invert in $\mu$, with $\beta$ fixed, and we assume without loss of generality that $a=\frac{1}{2}$ since this corresponds to the change of variables $t \rightarrow(2 a)^{2} t$. Now for large $\mu$ the transform is

$$
\mu^{-1}\left(1-e^{-\mu}\right) \sum_{n=0}^{\infty}\left(-\beta \frac{\left(1-e^{-\mu}\right)}{\mu}\right)^{n}
$$

and we recognize a sum of convolutions of the uniform density on $(0,1)$ times appropriate constants. The convolution formula of Feller, Vol. II, p. 27, yields as inversion (of the sum only) the expression 


$$
\begin{aligned}
\delta_{0}+\sum_{n=1}^{\infty} \frac{(-\beta)^{n}}{(n-1) !} \sum_{\nu=0}^{n}(-1)^{\nu}\left(\begin{array}{l}
n \\
\nu
\end{array}\right)(x-\nu)_{+}^{n-1} \\
=\delta_{0}+\sum_{\nu=0}^{\infty} \frac{(-1)^{\nu}}{\nu !} \sum_{n=\nu \vee 1}^{\infty}-\frac{\beta n}{(n-\nu) !}\left(-\beta(x-\nu)_{+}\right)^{n-1} \\
=\delta_{0}+\sum_{\nu=0}^{\infty} \frac{(-1)^{\nu}}{\nu !} \frac{d}{d x} \sum_{j=0}^{\infty} \frac{\left(-\beta(x-\nu)_{+}\right)^{j+\nu}}{j !} \\
=\delta_{0}+\frac{d}{d x} \sum_{v=0}^{[x]} \frac{(-1)^{\nu}}{\nu !}(\beta(x-\nu))^{\nu} \exp (-\beta(x-\nu)),
\end{aligned}
$$

where $\delta_{0}$ is the unit mass at 0 and the derivative is piecewise continuous. Convolving this with another uniform density yields for inversion of (2.3) simply

$$
-\beta^{-1} \frac{d}{d x} \sum_{\nu=0}^{[x]} \frac{(\beta(x-\nu))^{\nu}}{\nu !} e^{-\beta(x-\nu)}, \quad x>0 .
$$

Next, to find the inversion with $\sqrt{2 \lambda}$ in place of $\mu$, by Feller [5, XIII. 11, Exercise 13], we have the distribution of a product $X^{2} Y$ where $X$ has the above density in $x, Y$ has transform $\exp (-\sqrt{2 \lambda})$, and $(X, Y)$ has the product measure. The density of $Y$ being $(2 \pi)^{-1 / 2} y^{-3 / 2} \exp \left(-(2 y)^{-1}\right)$, this yields for the product the density in $t$

$$
\frac{-\beta^{-1}}{t \sqrt{2 \pi}} \int_{0}^{\infty} \sum_{\nu=0}^{[\sqrt{t / y}]} \frac{(\beta(\sqrt{t / y}-\nu))^{\nu}}{\nu !} e^{-\beta(\sqrt{t / y}-\nu)} \frac{d}{d y}\left(y^{-1 / 2} \exp -(2 y)^{-1}\right) d y,
$$

where we used integration by parts.

To invert in $\beta$, we observe that the terms $\beta^{\nu-1} \exp (-\beta(\sqrt{t / y})-\nu)$ correspond to the $\nu-1$ st derivatives at $\sqrt{t / y}-\nu$. Hence the integral of our density in $\alpha$ times a $C^{\infty}$-function $f(\alpha)$ with compact support in $(0, \infty)$, for fixed $t$, is given by

$$
\begin{aligned}
-\frac{1}{t \sqrt{2 \pi}} & \sum_{\nu=0}^{\infty} \frac{(-1)^{\nu-1}}{\nu !} \\
& \cdot \int_{0}^{t \nu^{-2}}\left(\sqrt{\frac{t}{y}}-\nu\right)^{\nu} f^{(\nu-1)}\left(\sqrt{\frac{t}{y}}-\nu\right) \frac{d}{d y}\left(y^{-1 / 2} e^{-(2 y)^{-1}}\right) d y
\end{aligned}
$$

where $f^{(-1)}$ is the indefinite integral from 0 . To eliminate the derivatives on $f$, we integrate by parts a corresponding number of times to get, after setting $\alpha=\sqrt{t / y}$ $-\nu$ in the $\nu$ th term,

$$
\frac{t^{-3 / 2}}{\sqrt{2 \pi}} \sum_{\nu=0}^{\infty} \frac{1}{\nu !} \int_{0}^{\infty} f(\alpha) \frac{d^{\nu-1}}{d \alpha^{\nu-1}}\left(\alpha^{\nu}\left(\frac{(\alpha+\nu)^{2}}{t}-1\right) \exp \left(-(2 t)^{-1}(\alpha+\nu)^{2}\right)\right) d \alpha
$$

It is clear ab initio that there is no $\alpha$-mass at 0 , and the reader will note that the above steps are reversible. Hence the distribution in $\alpha$ is indeed absolutely continuous, and by varying $f$ and checking the integral for $\nu=0$ the densiity can 
be written as in (2.2). The marginal density in $t$ follows by integration of the first two terms in $\alpha$.

It is now straightforward to give the solution of the problem cited in the introduction. We have

Theorem 2.3. The density in $\alpha>0$ of the local time at $a>0$ of the Brownian excursion of duration $l>0$ is

$$
2^{3 / 2} \pi^{5 / 2} a^{-3} l \int_{0}^{1} f_{2}\left(\frac{l}{2}\left(\frac{\pi}{a}\right)^{2}(1-t)\right) f\left(t l^{-1 / 2}, \alpha l^{-1 / 2}\right) d t
$$

where $f_{2}$ is given by (1.4) and $f$ by (2.2). There is an atom at 0 of magnitude $F_{2}(a)$.

Proof. Let $E_{l}(t)$ denote the excursion of duration $l$. By the scaling property of $W(t)$ we have $E_{l}(t) \equiv \sqrt{l} E_{1}\left(t l^{-1}\right)$, where $E_{1}$ is our scaled excursion. Then the local time at $a>0$ is

$$
\frac{d}{d a} \int_{0}^{l} I_{(-\infty, a)}\left(E_{l}(t)\right) d t \equiv \sqrt{l} l_{0}^{+}\left(a l^{-1 / 2}\right)
$$

and it suffices to change variables in the density of $l_{0}^{+}$. This is found by integrating the density of Corollary 1.3 times the conditional density in $\alpha$ given $t$ from (2.2). The factor $t^{-1 / 2}\left(1-\exp \left(-2 a^{2} t^{-1}\right)\right)$ cancels, and we have the result as stated.

REMARK. Noting that the density of Theorem 2.3 is a convolution in $t$, one can go back to the Laplace transforms and try to simplify the result by inversion of their product, but this inversion appears too complicated.

\section{REFERENCES}

1. K. L. Chung, Excursions in Brownian motion, Ark. Mat. 14 (1976), 155-177.

2. J. L. Doob, Heuristic approach to the Kolmogorov-Smirnov theorems, Ann. Math. Statist. 20 (1949), 393-403.

3. R. T. Durrett and D. L. Iglehart, Functionals of Brownian meander and Brownian excursion, Ann. Probability 5 (1977), 130-135.

4. R. T. Durrett, D. L. Iglehart and D. R. Miller, Weak convergence to Brownian meander and Brownian excursion, Ann. Probability 5 (1977), 117-129.

5. W. Feller, An introduction to probability theory and its applications, Vol. II, Wiley and Sons, Inc., New York, 1966.

6. R. K. Getoor and M. J. Sharpe, Excursions of Brownian motion and Bessel processes, Z. Wahrscheinlichkeitstheorie und Verw. Gebiete 47 (1979), 83-106.

7. K. Ito and H. P. McKean, Jr., Diffusion processes and their sample paths, Springer, Berlin, 1965.

8. H. P. McKean, Jr., Excursions of a non-singular diffusion, Z. Wahrscheinlichkeitstheorie und Verw. Gebiete 1 (1963), 230-239.

9. F. B. Knight, Brownian local times and taboo processes, Trans. Amer. Math. Soc. 143 (1969), $173-185$.

10. On the sojourn times of killed Brownian motion, (Séminaire de Probabilités XII 1978, University of Strasbourg), Lecture Notes in Math., vol. 649, Springer, Berlin and New York.

11. P. Lévy, Sur certains processus stochastiques homogènes, Compositio Math. 7 (1939), 283-339.

12. D. P. Kennedy, The distribution of the maximum Brownian excursion, J. Appl. Probability 13 (1976), 371-376.

13. G. E. Roberts and H. Kaufman, Table of Laplace transforms, W. B. Saunders Company, Philadelphia, 1966.

Department of Mathematics, University of Illinois at Urbana, Urbana-Champaign, Illinois 61801 